\title{
Benign nocturnal alternating hemiplegia of childhood
}

INSERM

\section{Source}

INSERM. (1999). Orphanet: an online rare disease and orphan drug data base. Benign nocturnal alternating hemiplegia of childhood. ORPHA:209973

Benign nocturnal alternating hemiplegia of childhood is a rare neurologic disease characterized by recurrent attacks of nocturnal screaming or crying followed or accompanied by unilateral or sometimes bilateral hemiplegia. Disorder is not associated with neurological or developmental impairments but may be associated with mild behavioral abnormalities. 